\title{
Quelques aspects des polyphonies instrumentales tule des Asurini du Moyen-Xingu
}

Some aspects of tule instrumental polyphony among the Asurini of the Middle Xingu

Jean-Pierre Estival

\section{OpenEdition}

Journals

Édition électronique

URL : http://journals.openedition.org/ethnomusicologie/1467

ISSN : 2235-7688

\section{Éditeur}

ADEM - Ateliers d'ethnomusicologie

\section{Édition imprimée}

Date de publication : 31 octobre 1993

Pagination : 163-179

ISBN : 2-8257-0485-7

ISSN : $1662-372 X$

Référence électronique

Jean-Pierre Estival, «Quelques aspects des polyphonies instrumentales tule des Asurini du MoyenXingu », Cahiers d'ethnomusicologie [En ligne], 6 | 1993, mis en ligne le 02 janvier 2012, consulté le 19 avril 2019. URL : http://journals.openedition.org/ethnomusicologie/1467

Ce document a été généré automatiquement le 19 avril 2019

Tous droits réservés 


\section{Quelques aspects des polyphonies instrumentales tule des Asurini du Moyen-Xingu}

Some aspects of tule instrumental polyphony among the Asurini of the Middle Xingu

Jean-Pierre Estival

Je remercie Pierre di Sciullo qui a réalisé les Fig. 1 et 13.

« Des voix distinctes qui sonnent séparément, mais harmonieusement et sonnent harmonieusement quoique séparément ». Gui d'Arezzo, Micrologus

1 C'est un constat fréquent pour les ethnomusicologues américanistes d'affirmer la méconnaissance que nous avons des mondes sonores des Amérindiens d'Amazonie et plus généralement des Basses Terres d'Amérique du Sud ${ }^{1}$ : sur plus d'une centaine de cultures originales encore vivantes, à peine plus d'une dizaine sont sérieusement documentées, et les trois-quarts demeurent totalement inconnues. Dans ce cadre consacré aux polyphonies, nous nous concentrerons sur la présentation et l'analyse de la production sonore des tule asurini. Cependant, notre position n'en demeure pas moins claire : si la musique forme système, c'est bien pourtant, dans ces sociétés, l'ensemble du complexe rituel qui donne le sens.

2 Les Asurini constituent aujourd'hui une société de soixante personnes vivant sur les marges orientales du cours moyen du fleuve Xingu, dans l'état de Pará, en Amazonie brésilienne. Les Asurini ont été approchés en 1971, dans une des régions du Brésil où les contacts entre les Amérindiens et les Brésiliens ont été les plus tardifs. Comme la plupart des Amérindiens d'Amazonie, les Asurini sont agriculteurs sur brûlis, chasseurs et pêcheurs. D'un point de vue sociologique, il s'agit d'une société sans institution hiérarchique, où la parenté joue un rôle central à travers les groupes résidentiels dans un 
jeu politique complexe ; les Asurini parlent une langue appartenant à la famille tupiguarani. Une dramatique chute démographique a suivi ce premier contact, laissant aujourd'hui seulement treize hommes adultes (quatorze en 1989 lors de la réalisation du tule dont nous allons parler ici). Paradoxalement, cette chute démographique a provoqué une très forte affirmation des valeurs culturelles de la société asurini, par l'intermédiaire en particulier d'une remarquable intensification des cures chamaniques. Près de la moitié des hommes adultes sont chamanes (paje), c'est-à-dire guérisseurs, mais aussi des hommes connaissant les dimensions cosmologiques dela culture asurini (Müller 1985, 1987). Les pratiques que nous appelons musicales sont centrées autour de trois rituels principaux : maraka, cure chamanique où le chant joue un rôle fondamental, tiwagawa, danse chantée liée à la guerre, et enfin tule. C'est un rituel d'une grande densité ethnographique, pendant lequel sont jouées les clarinettes tule au sein d'un orchestre alternant: chaque instrument ne produisant qu'une seule note, c'est le jeu polyphonique de plusieurs instruments regroupés en trois parties qui caractérise cette musique. Cette forme orchestrale est assez fréquente en Amazonie (Beaudet 1983 : 80-86).

\section{Le rituel tule : introduction}

Les Asurini commentent fort peu leurs pratiques rituelles : le récit mythique est une des seules voies d'accès direct à l'origine et au sens que la société asurini donne au tule. Voici une version du mythe d'origine des tule racontée ${ }^{3}$ par $\mathrm{K}$ (Homme de 65 ans, chamane) :

"C'est le serpent (mbaia) qui est le maitre du tule (tulejara). Il est invité pour faire la fête du tule par les Asurini; il avait forme humaine. Il y a de nombreux tule où dansent les animaux : jawoti tule, uruwu tule, kururu tule, urukuku tule, bien d'autres encore; il y a aussi le tule du kauj ${ }^{4}$ car les Asurini boivent du kauj pendant la fête; beaucoup de femmes dansent. Le serpent et les femmes dansent toute la nuit, et avant de terminer, le serpent emmène une femme Asurini : il l'emmène dans la terre, en dessous de la terre. Il a des relations sexuelles avec la femme, à l'aide de la clarinette, puis il s'endort. Il dort aussi lorsque le jour arrive, puisqu'il n'a pas dormi de la nuit. Le serpent a perdu sa forme humaine et a repris sa forme de serpent. La femme a alors pris peur en le voyant, elle s'est mise à crier. Le serpent, fâché par ces cris, est alors allé dans le fleuve, dans sa maison au fond du fleuve, en emmenant la femme avec lui. Pendant la fête, les Asurini ont appris le tule, et quand le serpent est parti, ils savaient jouer des clarinettes et faire le rituel. Aujourd'hui les Asurini continuent de faire le tule ».

Les informateurs décrivent les tule contemporains comme des réactualisations de ce mythe d'origine ${ }^{5}$. L'observation ethnographique de plusieurs tule, en 1978, 1980, 1982 et $1989^{6}$, nous permet de dégager les traits structurels suivants :

- Le tule est un rituel accompli pendant plusieurs mois, toujours pendant la saison des pluies (schématiquement de novembre à mai) ; son rythme n'est en général pas annuel.

- Le tule fait partie, avec d'autres rituels comme tauwa, boakara, kawara, d'un complexe «à géométrie variable »; il ne semble pas qu'il y ait de liens organiques nécessaires entre ces différents rituels qui furent étroitement associés par le passé7 ${ }^{7}$ En tous cas, pour le rituel de référence (1989), le tule seul a été accompli. Nous pouvons cependant partiellement expliquer cette simplification du complexe rituel par deux faits : d'une part, la situation démographique qui est vraiment catastrophique en ce qui concerne les hommes adultes, et d'autre part la topologie du village qui ne respecte plus la structure traditionnelle : les lieux 
rituels du village asurini n'existent plus, la communauté étant regroupée par la $\mathrm{FUNAI}^{8}$ selon un plan de « village rue ».

- D'un point de vue sociologique, le tule établit une partition du village asurini entre invitants pareara et invités tulywa, selon les groupes résidentiels ${ }^{9}$. Ces derniers constituent la base de l'organisation sociale actuelle (Müller, 1987).

- Les tulywa ont pour charge de jouer les clarinettes tule au sein d'un orchestre alternant; le maître des tule, tulejara, est responsable de l'ordonnancement rituel.

- Les pareara ont pour charge principale de fournir la bière (kauj) aux tulywa.

- Le rituel proprement dit est centré sur le jeu quotidien et éventuellement dansé, par les seuls hommes tulywa, de clarinettes appelées elles aussi tule. Plusieurs rites connexes sont adjoints, dont certains sont indispensables comme la préparation et la consommation du kau ¡ cérémoniel. Le jeu des tule en orchestre alternant n'a lieu que dans - ou devant, dans le cas de tule dansé - une maison cérémonielle nommée jangaiwa dans le village traditionnel (Figure 13).

6 Si l'on se réfère au mythe d'origine, le tule est un rituel lié aux alliances, le serpent invité séduisant les femmes du village. Ce rituel a été l'occasion de rencontres et d'échanges matrimoniaux entre groupes locaux dans la société traditionnelle, même s'il ne joue plus concrètement ce rôle dans la phase de vide démographique actuelle. Alors que le rituel tule participe d'une réaffirmation de l'ordre social via l'alliance - au moins en théorie -, force est de constater que les moments d'échanges entre pareara et tulywa ne sont que rarement liés à la pratique musicale, mais plutôt à la consommation du kauj cérémoniel, et aux pleurs rituels qui ponctuent les plus importantes libations. La plupart du temps, les tulywa jouent seuls, sans que les pareara assistent aux sessions.

7 Les sessions de jeu sont de deux types, auxquels sont liés deux répertoires différents, selon que les clarinettes sont jouées assises dans la maison rituelle ou dansées - parfois avec les femmes - sur l'aire cérémonielle. Les tulywa se réunissent une fois, deux fois ou trois fois par jour, au lever du soleil, à midi, au coucher du soleil ou la nuit ${ }^{10}$ pour interpréter une série de pièces dont chacune a un nom. Ce nom est souvent celui d'un animal - ceux qui dansèrent lors du tule primordial -, mais on a aussi le tule du kauj, de la pluie, du sexe féminin ou du fœetus : ces derniers sont à mettre en relation avec l'échange (le kauj cérémoniel) ou l'alliance. Examinons de plus près ces polyphonies instrumentales.

\section{Organologie et jeu des clarinettes tule}

8 Les tule sont des clarinettes idioglottes, de facture composite, à tuyau rapporté, et sans trou de jeu (Estival 1991 : 134), dont la coupe est donnée en Figure 1; chaque instrument ne peut produire qu'une seule note. Les Asurini distinguent quatre tule : tule towapey'i, tule mytera, tule ywyra et tule tahira. Les aspects sémantiques des noms de ces différents instruments sont complexes : une polysémie certaine s'y attache, que mes compétences linguistiques ne me permettent pas d'appréhender pleinement; les Asurini donnent couramment les équivalents suivants :

towapey' $i$ : « petit visage » (towape : « figure, visage », + i : diminutif.

mytera : « celui du milieu ».

ywyra: «bois », « frère cadet » (ego masculin).

tahira : «fils » (ego masculin).

9 Les porte-anches sont faits dans un tube de roseau assez durable. Un nœud ferme le porte-anche à son extrémité supérieure et, à quelques millimètres de ce nœud, une anche 
battante est détachée sur une longueur variant entre 58 et $70 \mathrm{~mm}$. L'anche voit sa vibration contrôlée par une ligature faite en fil de coton, qui, coulissant autour du tube, permet de déterminer la longueur de lamelle vibrante, et ainsi d'accorder l'instrument. Le porte-anche est fixé, à l'aide d'une bourre de coton, dans le nœud percé à cet effet dans le corps (tuyau) de l'instrument fait de bambou (Gadua superba). Les instruments towapey'i, mytera et ywyra ont des tuyaux de taille croissante, mais comparable. Les diamètres internes varient entre 25 et $50 \mathrm{~mm}$. On a les mesures moyennes de longueur: pour towapey'i : 901,5 mm ; pour mytera : $971 \mathrm{~mm}$; pour ywyra : $1075 \mathrm{~mm}$. On retiendra que ces longueurs peuvent varier de manière significative au sein d'une même classe.

Fig. 1 : Coupe du tule

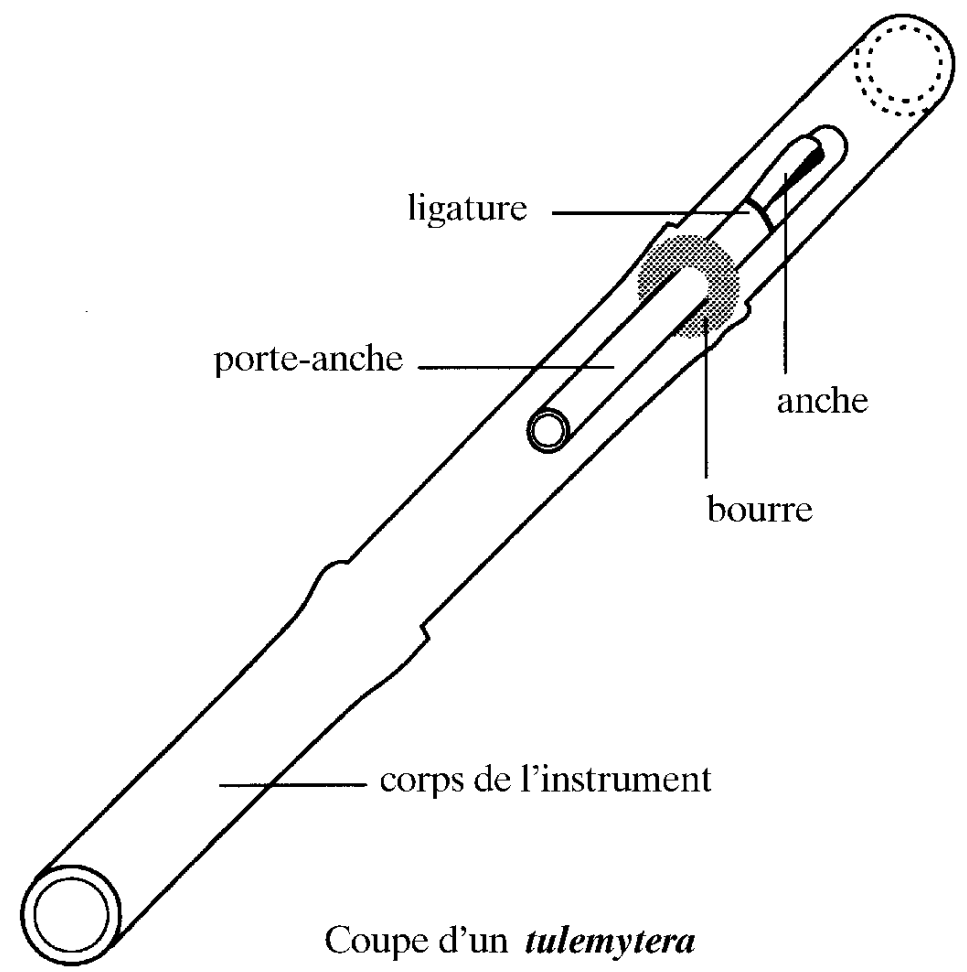

L'instrument tahira est beaucoup plus court : un seul exemplaire a été utilisé en 1989 ; sa longueur était de $540 \mathrm{~mm}$ et son diamètre de $19 \mathrm{~mm}$. Cet instrument, au rôle et au timbre particuliers, ne se distingue pas par sa facture des autres tule. Le tulepy qui joue la partie ywyra joue aussi l'instrument tahira. Les tule sont décorés par de la peinture et/ou des plumes : pour les towapey'i, mytera et ywyra, ces motifs sont des losanges, pour tahira, trois cercles peints ornent la partie inférieure de l'instrument. L'ensemble de ces motifs est spécifique des tule et n'apparaît pas ailleurs dans le très riche art graphique des Asurini. Les instruments sont joués debout ou assis, et tenus souvent par la seule main gauche placée à mi-hauteur du tuyau (Figure 2). Les instrumentistes se positionnent en demicercle (tule joué assis), ou en file indienne (tule dansé), toujours dans l'ordre suivant : en tête le tulejara et son towapey'i, puis le (les) tulepy qui joue(nt) mytera, et enfin le (les) tulepy qui joue(nt) ywyra et tahira. 
Figure 3

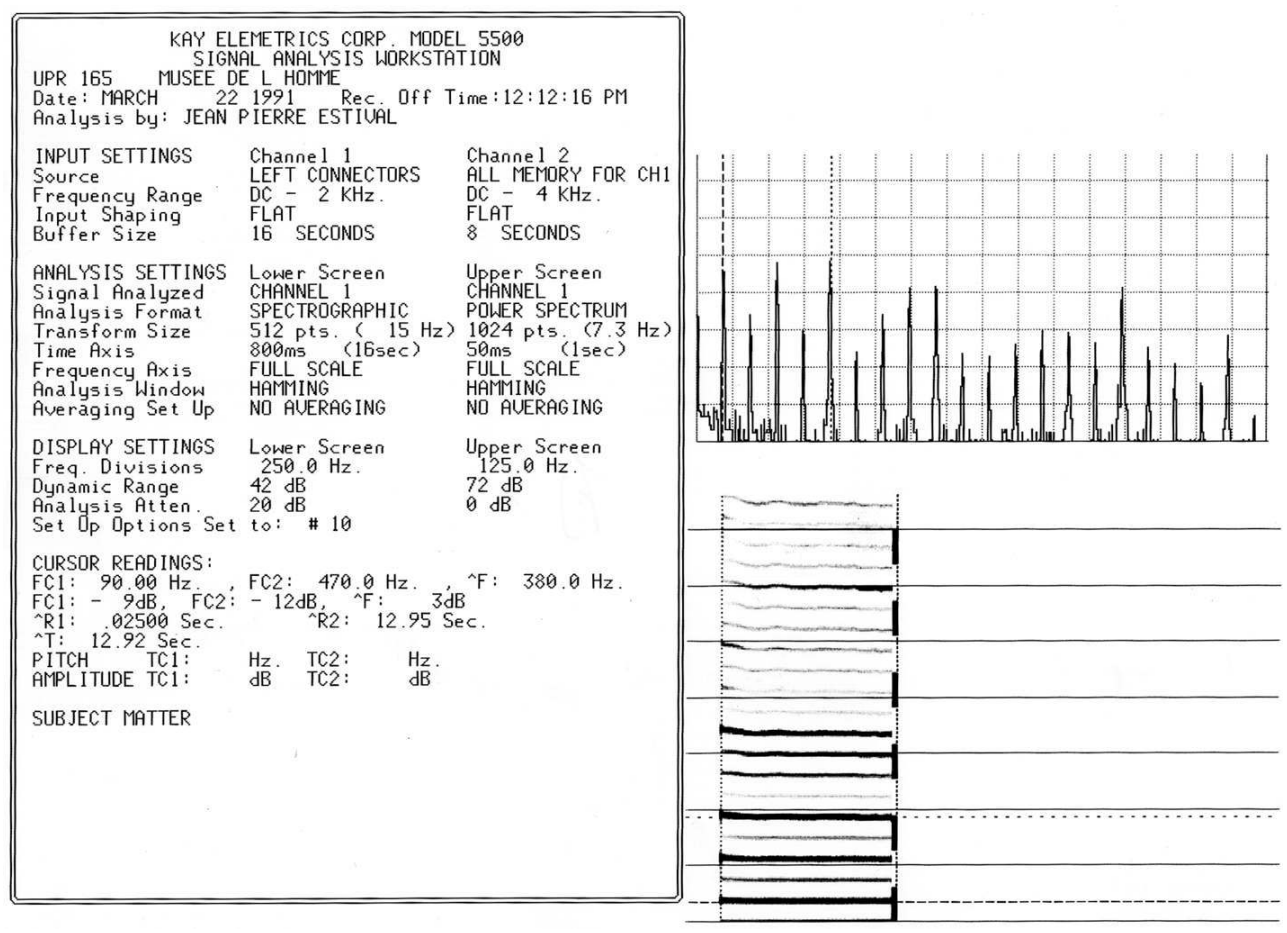

Figure 4

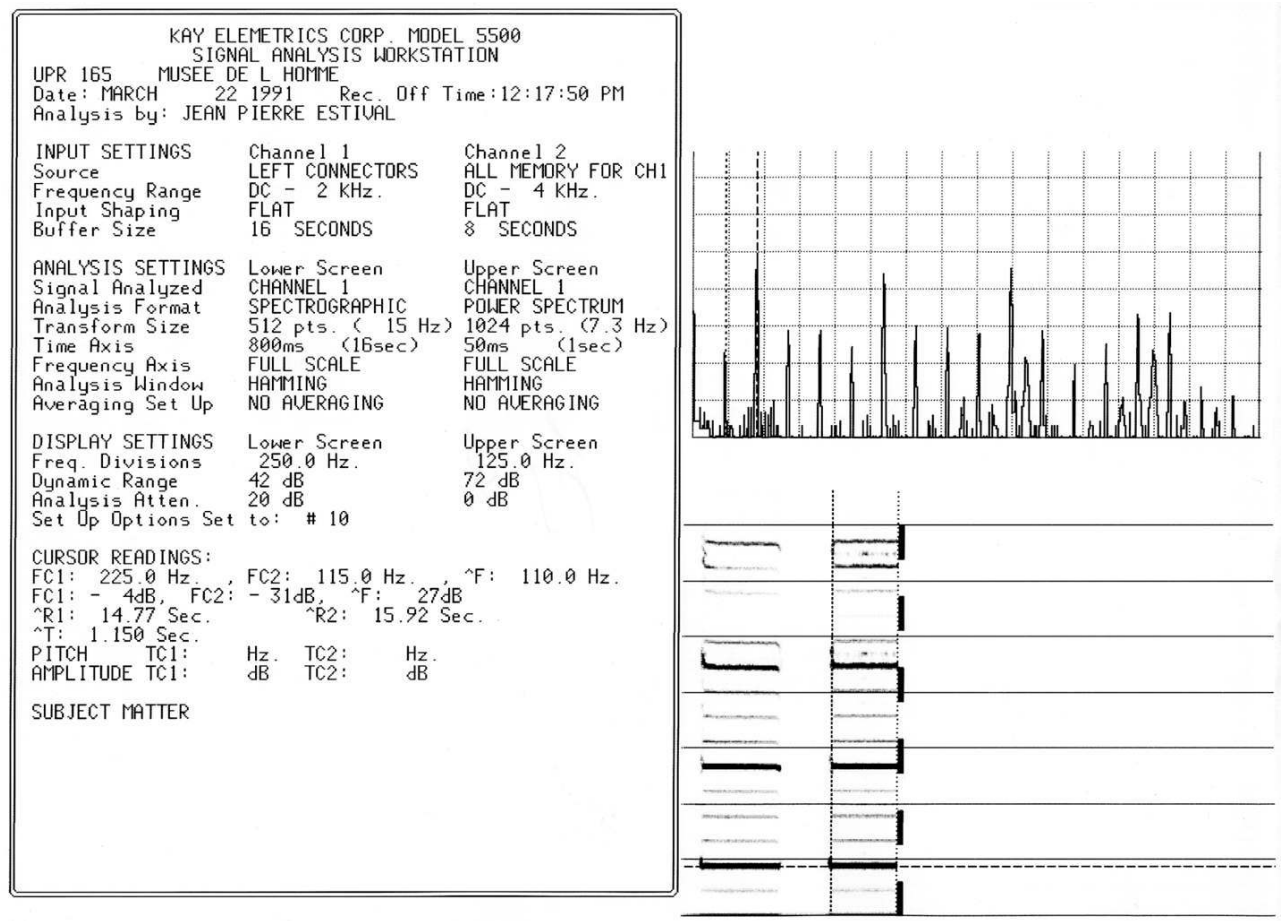


Fig. 2 : Pièce tule jouée assise

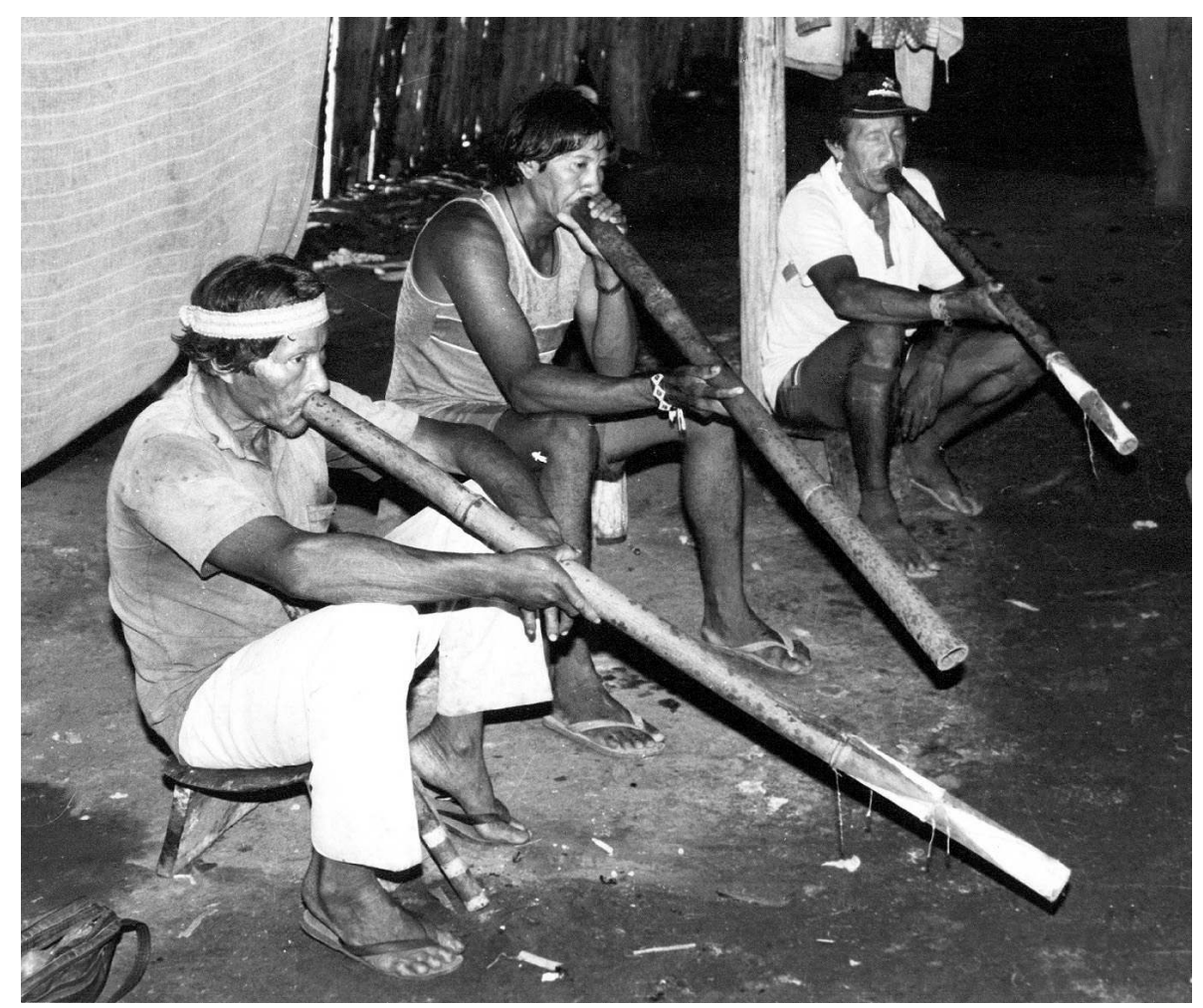

11 La technique instrumentale proprement dite est réduite à sa plus simple expression : il s'agit de souffler au bon moment, sans pression excessive ${ }^{11}$. Il arrive bien sûr que plus de trois tulepy jouent simultanément: alors, ce sont les voix mytera et ywyra qui sont doublées ou triplées (voir aussi p. 167).

\section{Les timbres des tule; les échelles}

L'analyse sonagraphique montre que l'on peut distinguer, parmi les tule, deux classes: towapey'i, mytera, ywyra d'une part, tahira d'autre part.

Les trois premiers instruments, qui diffèrent légèrement par leur taille, et surtout par la hauteur de leurs fondamentales respectives, possèdent des timbres assez homogènes : ils se comportent nettement comme des clarinettes, avec une fondamentale et ses premiers harmoniques impairs renforcés (sonagramme de la Figure 3). La différence de timbre apparaît dans l'intensité des harmoniques de rang $>5$ et des zones de formant qui en résultent. Ces harmoniques sont nombreux (plus de 60 en général), et remplissent le spectre, avec des intensités variables, jusqu'à plus de $4 \mathrm{KHz}$, ce qui est considérable pour des instruments dont les sons fondamentaux ont une fréquence de l'ordre de $100 \mathrm{~Hz}$.

L'instrument tahira, beaucoup plus petit, a un timbre fort différent: fondamentale faible, voire très faible quand on souffle fort, et spectre assez instable (sonagramme de la figure 4). De surcroît, cette clarinette renforce le second harmonique, ce qui est étonnant pour cette famille d'instruments ${ }^{12}$ : cela prouve en fait que le tuyau réagit largement comme un résonateur et que c'est la petite clarinette idioglotte qui est acoustiquement le système fort. Les longueurs de ce porte-anche sont de même ordre de grandeur pour towapey'i et tahira, donc les fondamentaux sont de fréquences voisines. Le tuyau de towapey'i permet 
de renforcer, par sa fréquence propre, le son fondamental de l'instrument : il y a accord entre le porte-anche et le tuyau. Le tuyau de tahira, de moitié moins long que celui de towapey'i, a donc une fréquence propre qui renforce le second harmonique produit par le porte-anche (comparable à celui de towapey'i) : on peut ainsi expliquer la prégnance de ce second harmonique dans le timbre de tahira.

L'accord des instruments fait l'objet de peu d'attention, si ce n'est pour la qualité du timbre et la facilité d'émission. Lorsque les parties sont doublées, les instruments ne sont pas accordés entre eux. Les intervalles entre les instruments sont variables, et le fait le plus important est le maintien de la décroissance des hauteurs entre towapey'i, mytera et $y w y \mathrm{ra}^{13}$. Nous avons effectué, à l'aide du DPS sonagraph, des mesures de fondamentaux (ce qui a un sens, car les tule sont des instruments harmoniques ${ }^{14}$ ) sur les instruments qui venaient d'être réaccordés ${ }^{15}$. Après conversion des fréquences en hauteurs absolues (nom de la note - n Cents), nous avons les données dans la figure 5 , et les intervalles entre les trois parties principales dans la figure 6.

On remarquera, en ce qui concerne tahira, que le fondamental, de faible dynamique, est de hauteur comparable à celui de towapey'i, mais que l'accord n'est pas réalisé entre les deux instruments : tahira sonne « à peu près » à l'octave (voir plus haut) de towapey'i, mais on a parfois (jawara) des écarts très importants.

La valeur moyenne des intervalles entre towapey'i et mytera est de 215 Cents (avec un écart-type de 46 Cents), entre mytera et ywyra de 191 Cents (avec un écart-type de 21 Cents), et la valeur moyenne de l'ambitus entre towapey'i et ywyra est de 406 Cents (avec un écart-type de 47 Cents). Retenons en tous cas que la précision des échelles n'est pas une dimension essentielle pour le tule asurini, au vu de la labilité du système tel que l'on a pu l'observer.

\section{Syntaxe musicale : segmentation, interactions avec le geste chorégraphique}

18 Nous nous limiterons aux problèmes de description analytique des pièces tule. Rappelons qu'il n'y a pas, en général, unicité de la segmentation d'une pièce musicale en utilisant le critère de répétition, largement utilisé comme paradigme analytique (Rouget 1961, Ruwet 1972 et bien d'autres). Montrons-le. 


\begin{tabular}{|c|c|c|c|c|c|c|c|c|}
\hline $\begin{array}{l}\text { Nom } \\
\text { tule }\end{array}$ & kaul & jawara & tamádua & parati & jacu & tûga & tamádua & kara \\
\hline \multirow[t]{2}{*}{ To } & la2. & la2 & la2 & $1 \mathrm{a} 2$ & la2 & 132 & la2 & la2 2 \\
\hline & -10 & -40 & -30 & -30 & -30 & -96 & -40 & -40 \\
\hline \multirow[t]{2}{*}{ My } & sol2 & sol2 & sol2 & sol2 & sol2 & sol2 & sol2 & sol2 \\
\hline & -42 & -31 & -31 & -31 & +2 & -31 & -20 & -65 \\
\hline \multirow[t]{2}{*}{$Y w$} & $\mathrm{faz}$ & $\mathrm{faz}$ & $\mathrm{faz}$ & $\mathrm{faz}$ & $f a 2$ & $\mathrm{faz}$ & $\mathrm{fa2}$ & $\mathrm{faz}$ \\
\hline & -9 & -9 & -21 & -21 & -33 & -33 & -21 & -21 \\
\hline \multirow[t]{2}{*}{$\mathrm{Ta}$} & ré\# & la2 & la2 & la2 & la2 & la2 & la2 & \\
\hline & -47 & -64 & -80 & -16 & -9 & -40 & & \\
\hline \multicolumn{9}{|c|}{ Agoure 5.} \\
\hline \multicolumn{9}{|c|}{ Intervalles en Cents } \\
\hline $\begin{array}{l}\mathrm{N} \\
\text { tule }\end{array}$ & kaul & jawara & tamadua & $\stackrel{0}{\text { parati }}$ & jacu & tûga & tamádua & $\underset{\text { kara }}{m}$ \\
\hline$T$ & & & & 0 & & & & l \\
\hline My & 232 & 191 & 201 & 201 & 168 & 327 & 180 & 225 \\
\hline M & & & & y & & & & I \\
\hline$Y w$ & 177 & 178 & 190 & 190 & 235 & 202 & 201 & 156 \\
\hline$T$ & & & & 0 & & & & $I$ \\
\hline$Y_{w}$ & 409 & 369 & 391 & 391 & 403 & 529 & 381 & 381 \\
\hline
\end{tabular}

Figure 6.

Par exemple, soit une suite de symboles où $\mathrm{A}, \mathrm{B}, \mathrm{C}, \mathrm{D}, \mathrm{E}$ représentent des événements sonores discrets ${ }^{16}$ :

$\mathrm{S}=[\mathrm{ABCABCDEABCDE}]$.

On peut segmenter :

Seg1 $=[\mathrm{ABC}] /[\mathrm{ABC}] /[\mathrm{DE}] /[\mathrm{ABC}] /[\mathrm{DE}]$;

ou bien :

Seg2 $=[\mathrm{ABC}] /[\mathrm{ABCDE}] /[\mathrm{ABCDE}]$,

produisant ainsi des formules différentes, bien que $\mathrm{S} 2$ soit dans cet exemple réductible à S1, c'est-à-dire qu'en re-segmentant S2 on peut obtenir S1.

Mais prenons un autre exemple plus intéressant :

$\mathrm{S}^{\prime}=[\mathrm{ABCDEBCDABCFABCD}]$.

On peut segmenter :

Seg1' $=[\mathrm{A}] /[\mathrm{BCD}] /[\mathrm{E}] /[\mathrm{BCD}] /[\mathrm{A}] /[\mathrm{BCF}] /[\mathrm{A}] /[\mathrm{BCD}]$

en privilégiant $[\mathrm{BCD}]$ comme plus longue chaîne récurrente, ou encore :

Seg2' $=[\mathrm{ABC}] /[\mathrm{D}] /[\mathrm{EBC}] /[\mathrm{D}] /[\mathrm{ABC}] /[\mathrm{F}] /[\mathrm{ABC}] /[\mathrm{D}]$

en privilégiant $[\mathrm{ABC}]$ comme plus longue chaîne récurrente. Dans ce cas, les segmentations Seg1' et Seg2' sont irréductibles l'une à l'autre, et rien ne permet de décider, sans plus d'information, que l'une des segmentations soit «meilleure" que l'autre. Autrement dit, il n'y a pas, en général, unicité de la segmentation.

20 Ainsi, pour la construction d'un système de règles ${ }^{17}$ générant des pièces synthétiques (Johnson-Laird 1991, pour un travail abouti), nous devons avoir des hypothèses supplémentaires qui permettent de rendre explicites et systématiques les principes de segmentation ${ }^{18}$.

21 Revenons au tule. La musique des tule est jouée sur une pulsation isochrone (environ 80 pulsations/min. pour les pièces jouées assis, 60 pour les pièces dansées), matérialisée par les mouvements de haut en bas qu'imprime le tulejara à son instrument. Une première 
segmentation ne pose pas vraiment de problème : les pièces tule sont organisées selon la macro-structure suivante :

formule introductive - structure périodique jouée $n$ fois à l'identique - formule terminale.

Pour analyser de plus près la structure des tule, nous avons besoin d'une transcription graphique, d'un type déjà utilisé par Beaudet (1983) et Estival (1991) pour des musiques comparables: les parties sont écrites ligne par ligne ${ }^{19}$, la croche étant l'unité étalon matérialisant la pulsation.

Les formules introductives ont deux caractéristiques: d'une part leur rôle d'initiation et de stabilisation du hoquet par le calage de la pulsation, d'autre part le jeu en alternance, dans la plupart des pièces, de towapey'i et de tahira. Comme ces deux instruments ont un fondamental de hauteur presque identique, mais des spectres très différents, on pourrait appeler « opposition de timbre » cette alternance (I sur Figure 11).

La fin des pièces est marquée par un geste du tulejara qui se traduit par une note émise par le tulepy qui joue tahira: cette fin peut intervenir - au vu des observations sur l'ensemble du corpus -, en un point quelconque de la période.

Comme nous l'avons vu dans les exemples théoriques, le problème de la segmentation à l'intérieur des périodes suppose des hypothèses plus fortes que le simple critère de répétition. J'avais empiriquement constaté, pendant le rituel, que deux types d'organisation sonores pouvaient être produits par l'orchestre des tule: d'une part des mélodies produites par l'alternance des parties, et d'autre part des sortes de clusters produits par le jeu simultané des trois parties. De plus, les Asurini semblaient attacher une grande importance à ce jeu simultané des parties ${ }^{20}$; on peut donc envisager de découper la structure périodique en formules dont l'organisation interne serait consonante ou alternante.

La première organisation, consonante, consiste en un jeu simultané des trois instruments selon un patron rythmique fixe calé sur la pulsation, auquel j'inclus une dernière pulsation où les parties $\mathrm{M}$ et $\mathrm{T}$ sont souvent élidées; ce dernier temps fonctionnant comme le marqueur de la fin de la formule consonante.

La seconde organisation, alternante, consiste en un jeu mélodique en hoquet à une voix contre deux simultanées, ou bien à trois voix séparées. Voici les descriptions syntaxiques de quelques pièces, les premières d'un même groupe de tule joués assis par trois musiciens :

urukuku tule (figure 7)

28 La formule A est alternante sur 8 pulsations; elle est suivie d'une formule A' qui ne se différencie de A que par la dernière pulsation, jouée par Y (ywyra) en A', et par M (mytera) et $\mathrm{T}$ (towapey'i) en $\mathrm{A}$; la formule B est consonante et de longueur 8 ; la formule $\mathrm{C}$ est consonante, de longueur 4 ; C' est formée d'une formule consonante de longueur 3 suivie d'une pulsation jouée par $\mathrm{M}$ et $\mathrm{T}$. En comparant respectivement les derniers temps (pulsation) de A et A' d'une part, C' et C d'autre part, on constate la même opposition de fin de formule : $\mathrm{T}$ et $\mathrm{M}$ contre $\mathrm{Y}$. La période est de longueur 32 [A A' B C C'], régulièrement répétée. 
Figure 7
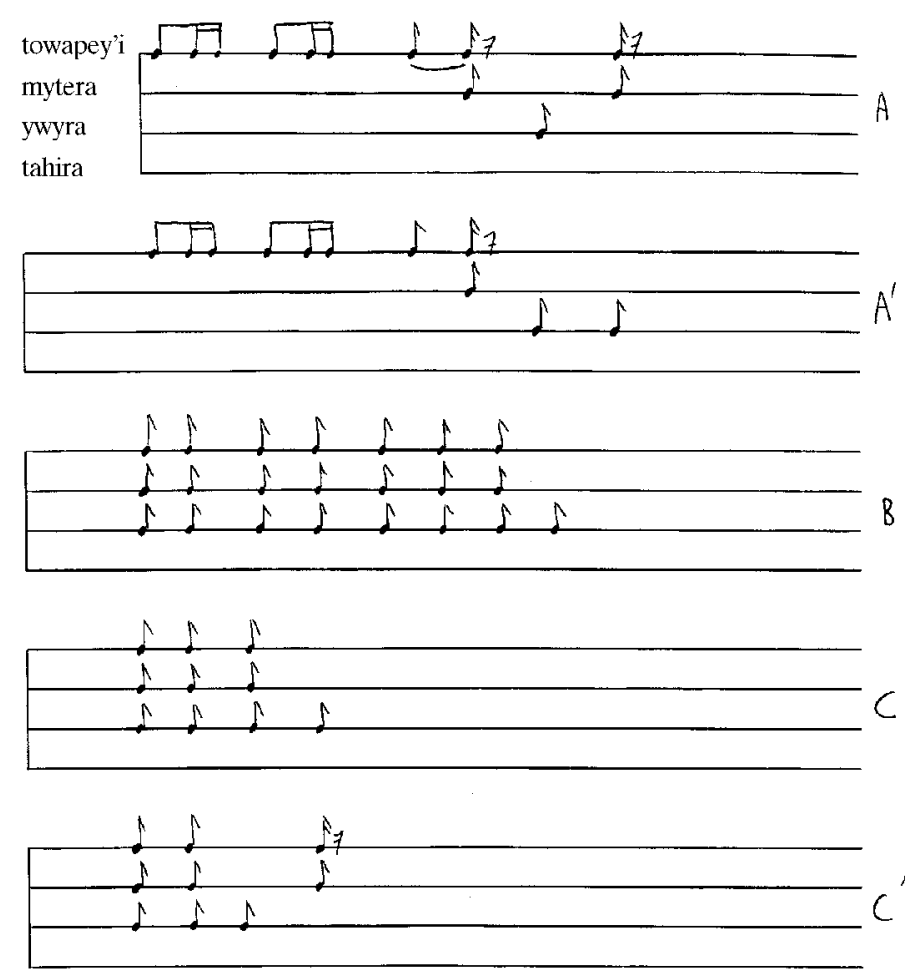

urukuku tule (figure 8) :

La formule A est alternante de longueur 6 ; B est le doublement d'une formule consonante sur 2 temps, soit 4 pulsations; $C$, enfin est une formule consonante sur 8 pulsations. La longueur de la période est 26 [A B C C]. 
Figure 8
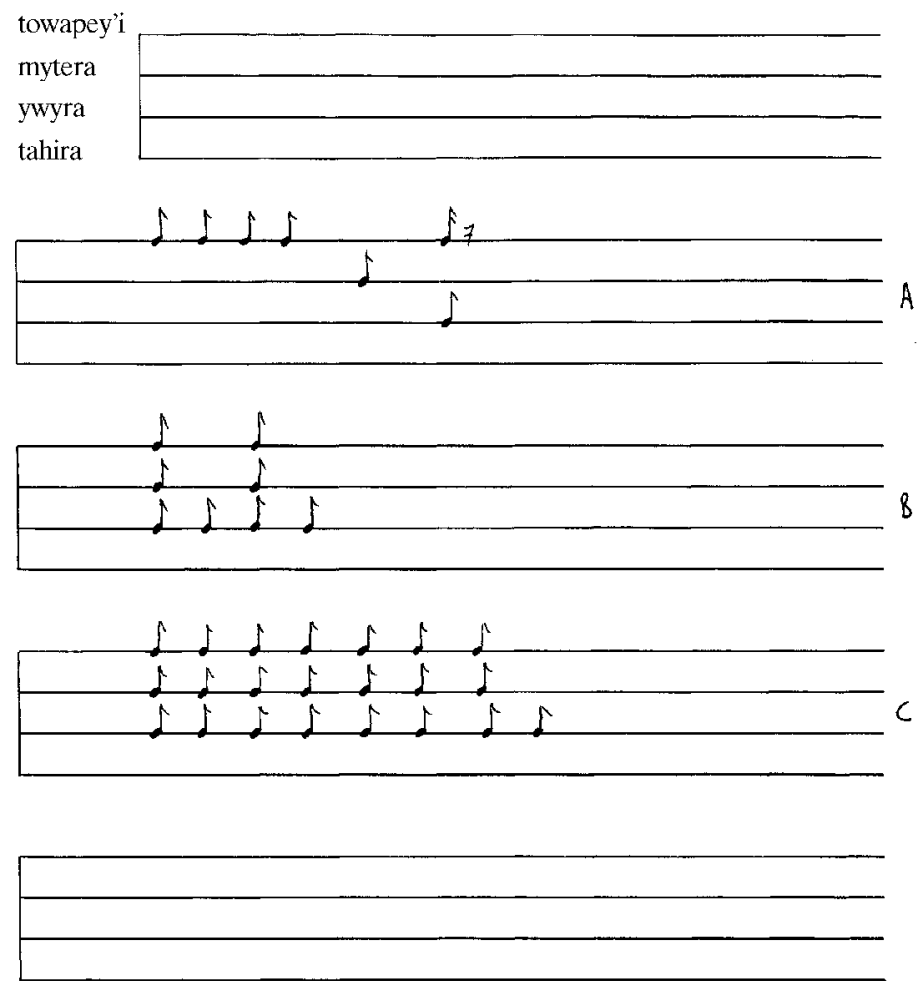

urukuku tule (figure 9) :

La formule A est alternante de longueur 9 ; la formule B est consonante sur 4 temps, avec un monnayage rythmique sur la dernière pulsation ; $B$ ' ne diffère de $B$ que sur sa dernière pulsation. La période est de longueur 30 [A A B B B']. 
Figure 9
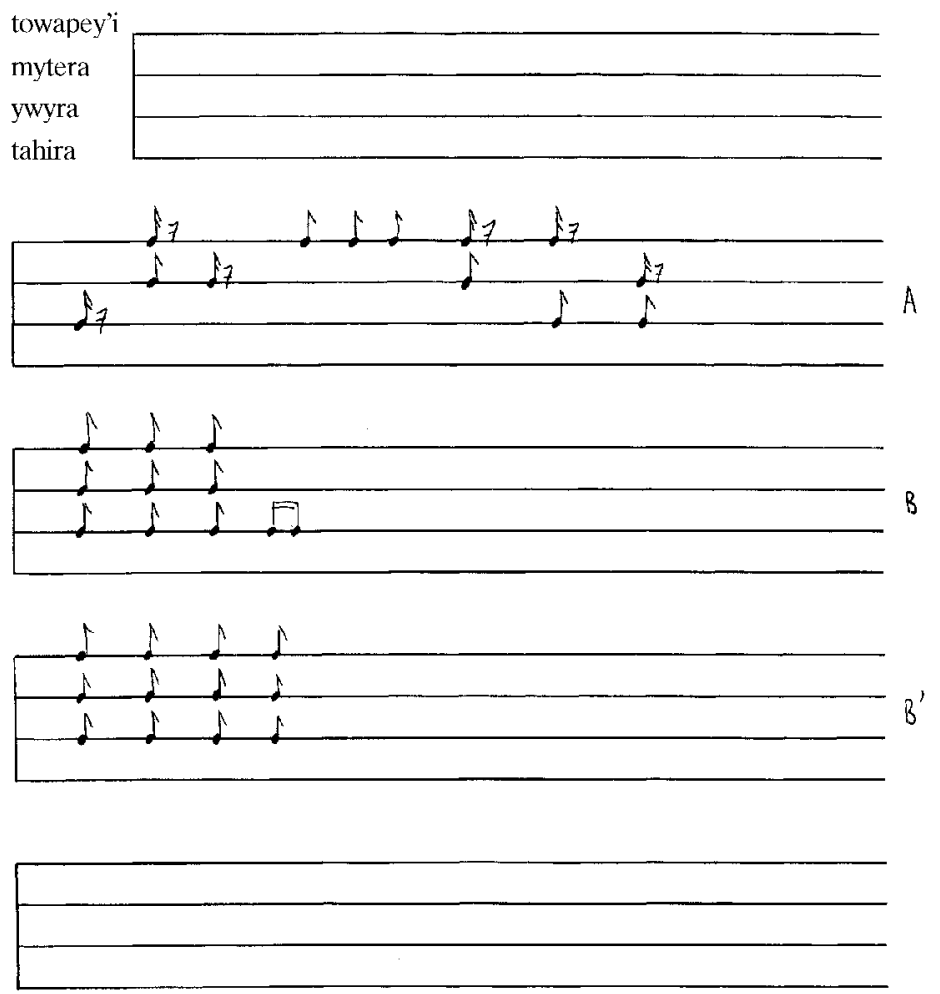

urukuku tule (figure 10):

31 La formule A est alternante sur 4 pulsations ; B et B' sont consonantes et de longueur 4, C de longueur 3, D est aussi une formule consonante, comme D' qui est de longueur 5. La période est de longueur 32, [A B A B' C D C D']. On remarque que des groupements peuvent être faits, qui pourraient donner lieu à une autre segmentation :

$A B: 4+48$
$A^{\prime} 4+48$
$C^{2} 3+58$
$C^{\prime} 3+58$ 
Figure 10

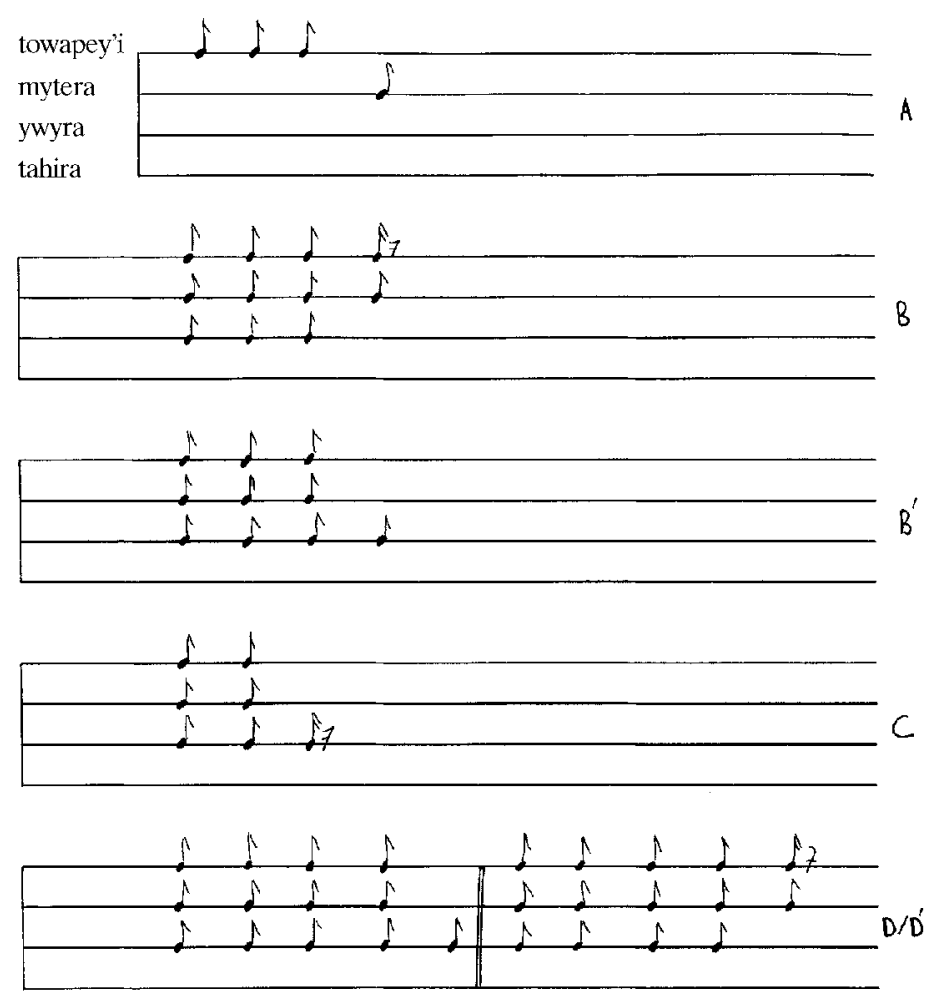

32 Si l'on compare la structure de ce tule avec celle du tule transcrit sur la Figure 7, on a la même longueur de période, mais une économie des formules différente, quoique toujours réductible à un découpage sur 8 pulsations.

Puis tamãdua tule (figure 11):

La formule A est alternante sur 4 pulsations ; les formules B et B' sont consonantes, sur 4 temps; la formule $C$, enfin, est constituée d'une formule consonante sur 6. Du fait du caractère périodique de la structure des tule, la formule $\mathrm{D}$ aurait pu être attachée au début de $A$ : cependant $A$ apparaît seule en début de pièce, ce qui m'a fait préférer cette segmentation. La période est de longueur 24 [A B A B' C], régulière. On remarquera que, bien que 24 soit un multiple de 8 , une segmentation en trois parties égales n'est pas réductible à des parties consonantes et alternantes. 
Figure 11
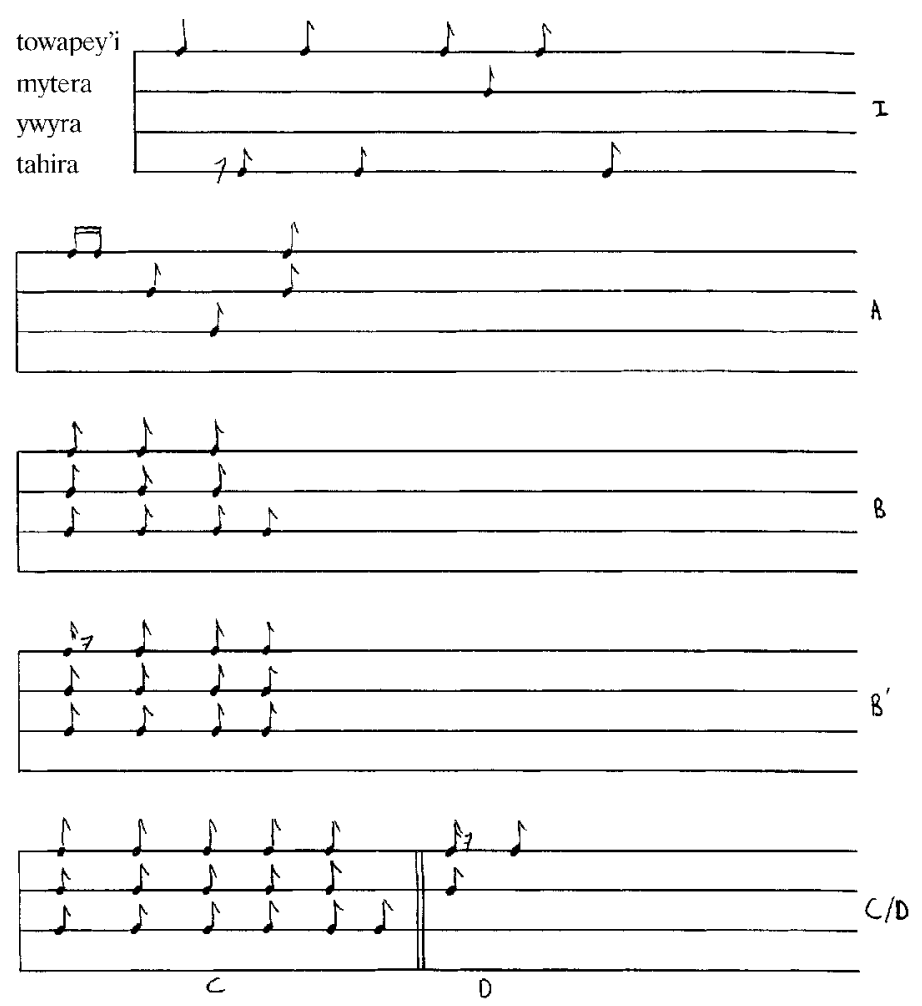

La syntaxe musicale, dans le cas de pièces dansées, n'est pas indépendante de la chorégraphie qui l'accompagne. Nous prendrons un exemple, parmi d'autres, où la danse a pu être documentée (sur le terrain, par une description juste à la suite de l'enregistrement) :

karowaru tule (transcription musicale Figure 12)

Il s'agit d'un tule dansé en rond, avec figures ; la valeur de la pulsation est ici la noire. La formule A, alternante, se développe sur 6 pulsations ; la formule $\mathrm{C}$ est aussi alternante sur 6 pulsations ; 4 formules consonantes sont jouées, sur 10, 8, 4 pulsations, respectivement B, B' (comme B moins les deux premiers temps), B". 
Figure 12

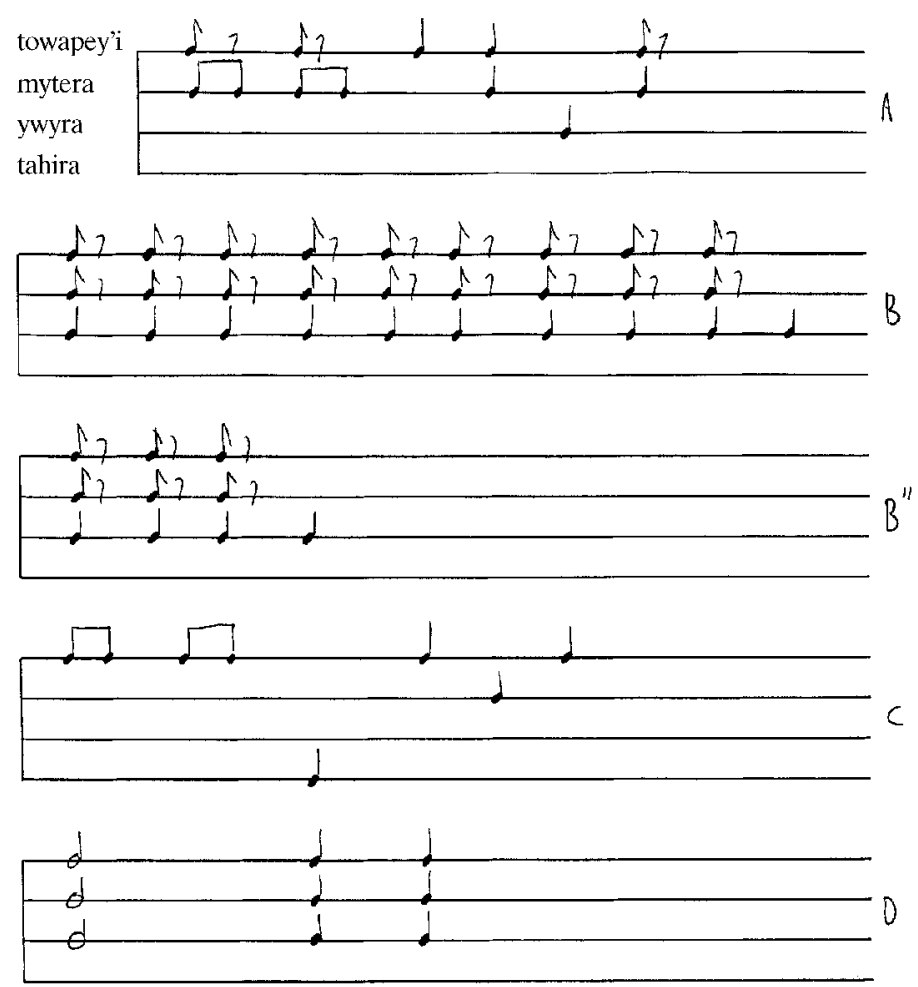
chorégraphie, les musiciens étant eux-mêmes danseurs. On peut, après avoir décrit la danse (Figure 13) expliquer la présence des formules D et C dans la description
syntaxique. syntaxique. Les musiciens jouent le début de la pièce en restant sur place, se balançant d'une jambe sur l'autre; depuis ce point de départ, les tulepy font ensemble un demi-tour. Arrivés à ce point diamétralement opposé, T (mytera) et MO (ywyra et tahira) restent à danser sur place, frappant avec vigueur le sol de leur pied droit; MW (mytera) et A (tulejara) partent pendant ce temps vers le centre du cercle, selon deux cordes parallèles, en zigzaguant de manière régulière. Une fois arrivés à la hauteur du centre, ils reviennent vers le $\mathrm{T}$ et $\mathrm{MO}$ de la même manière. Les quatre musiciens/danseurs partent alors deux par deux, TR avec MO, A avec MW, en suivant les mêmes zigzags que précédemment vers le centre du cercle. C'est à ce moment là que la formule $\mathrm{D}$ est jouée car les deux couples reviennent décalés sur la circonférence du cercle. Les 4 tulepy reprennent leur danse conjointe afin de compléter leur demi-tour.

Dans cet exemple, c'est le décalage de certains des danseurs qui a provoqué le jeu de la formule $\mathrm{D}$; celle-ci, consonante, se prête bien à ce rattrapage, par la simplicité de son énonciation. La formule $C$, jouée juste après, comprend sur les $2^{\mathrm{e}}, 3^{\mathrm{e}}$ et $4^{\mathrm{e}}$ pulsations le jeu en « opposition de timbre » de towapey'i et tahira : on a vu que cette organisation musicale apparaissait en général en début de pièce. Tout se passe comme si, après un événement fortuit, les musiciens reprenaient la pièce en remplaçant A par une formule marquant, à 
la fois techniquement et aussi symboliquement, la reprise correcte du morceau. Le système qui est à la base de l'organisation syntaxique des tule est suffisamment souple pour intégrer sur le champ un élément syntaxique nouveau, rendu nécessaire par un « accident » chorégraphique. Il est probable que la gestion, pour les trois tulepy, de ces irrégularités a été menée par des gestes ou signes appropriés du tulejara; ignorant les principes musicaux lors de l'observation du rituel, je n'ai malheureusement pas noté précisément la gestuelle des musiciens à ce moment là. Cela nous conforte dans l'idée que le découpage en formules telles que nous l'avons proposé est bien pertinent (bien que non unique): $\mathrm{D}$ est une formule consonante, quoiqu'inhabituelle, et $\mathrm{C}$ est alternante, et compatible avec A (même longueur au sein du cycle).

Figure 13 : Danse karowaru

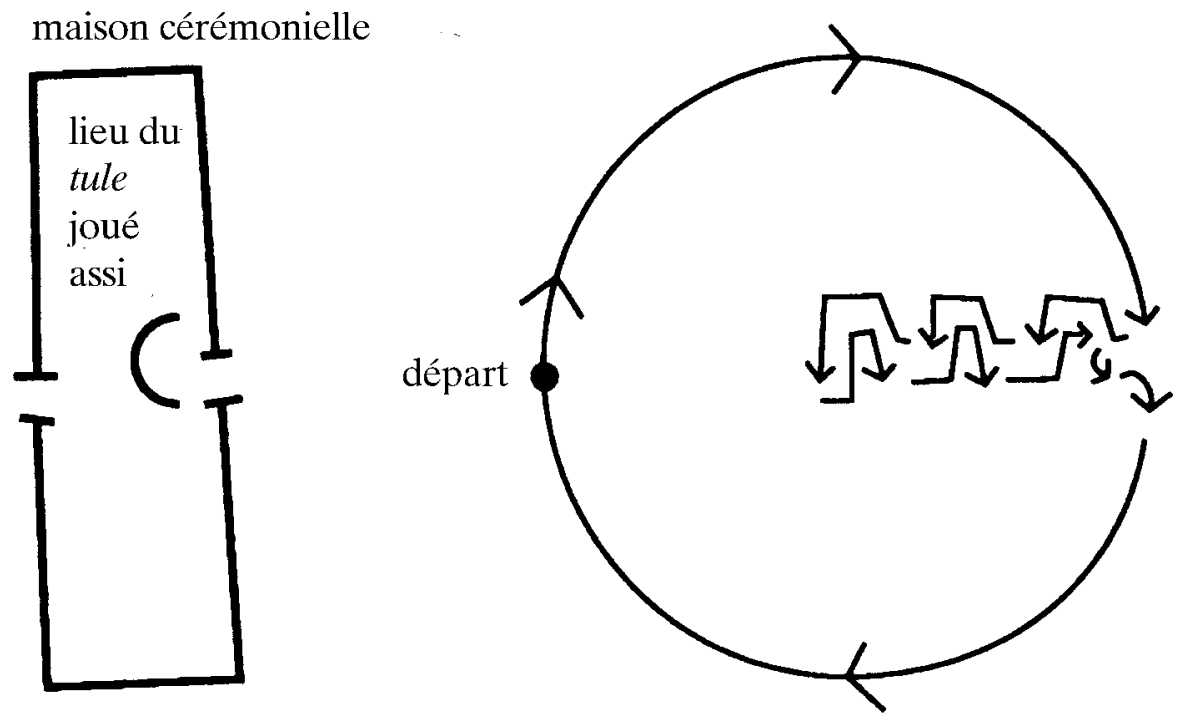

karowaru tule: chorégraphie

41 Nous pouvons inférer, à partir de ces quelques exemples ${ }^{21}$, un ensemble de caractéristiques permettant de définir, de façon univoque, l'organisation syntaxique et le déroulement dans le temps des pièces tule:

1. la musique des tule est une musique à trois parties fondamentales, plus une intervenant essentiellement en début et en fin de pièce ;

2. la musique des tule est jouée sur une pulsation isochrone ;

3. une première segmentation des pièces met à jour des périodes répétées pendant le déroulement de la pièce ;

4. ces périodes sont de longueur variable, entre 16 et 40 pulsations, avec souvent 32 pulsations ;

5. les périodes sont segmentables en formules alternantes et formules consonantes; toutes les pièces tule comprennent les deux types de formule;

6. ces formules sont de longueurs diverses, sans qu'il y ait nécessité de segmentation régulière au sein de la période ;

7. la dernière pulsation des formules alternantes, comme celle des formules consonantes est en général jouée soit par mytera, soit par ywyra, avec éventuellement une ponctuation de towapey'i; 
8. le mouvement mélodique des formules alternantes est en général descendant ;

9. le système de concaténation de formules est contrôlé par la longueur de la période, mais son principe même permet, quand l'exécution rituelle l'impose, de concaténer des formules supplémentaires ou de remplacement ;

10. pour le début des pièces, on a une mise en place du hoquet intégrant l'opposition towapey'i/ tahira ;

11. pour la fin des pièces, la période peut être interrompue sans qu'elle soit achevée ; la fin est marquée par le jeu de tahira.

En définitive, on peut considérer, en tenant compte des caractéristiques énoncées cidessus, qu'une pièce de tule est syntactiquement décrite par :

1. la longueur de sa période ;

2. une ou plusieurs formules alternantes au contour mélodique et de longueur propre ;

3. une ou plusieurs formules consonantes ;

4. la façon dont ces différentes formules sont concaténées au sein de la période.

\section{Conclusion}

Cette description analytique d'une musique polyphonique amazonienne ne concerne que certains aspects formels de la production sonore des tule. Nous ne pouvons ici - ce serait l'objet d'un article à part entière - qu'évoquer quelques-unes des relations qu'entretiennent ces polyphonies et leur structure avec la culture qui les a produites. Je me contenterai donc de quelques remarques.

Tout d'abord, et là comme ailleurs, le moment musical relève d'un découpage du temps lié à de fortes contraintes (rythme et syntaxe), alors que l'ensemble du rituel se déroule selon un temps long et peu contraignant. Cette opposition montre le rôle de marqueur et de ponctuation assigné à la performance des pièces tule: marquage particulièrement signifié par l'intervention de tahira, en début et à la fin des pièces.

Des homologies formelles existent entre la structure des pièces et celle de l'art graphique tayngawa des Asurini. De même, certains mouvements chorégraphiques (le motif esquissé par exemple dans karowaru tule) existent comme motif graphique (Müller 1987 : 279-367).

La polyphonie musicale des clarinettes semble être intégrée, dans un rituel polymorphe concernant des acteurs différents (pareara/tulywa), au sein d'une plus vaste polyphonie sociale où chacune des voix participe de la régulation de la vie des hommes et sans doute des rapports au monde (on ne connaît que peu de choses de la cosmologie asurini). La musique apparaîtrait alors, mais cela n'est pas très original, comme un médiateur des hommes entre eux, et des hommes avec le monde des esprits.

Au niveau régional (Moyen-Xingu/Bas-Iriri), on constate l'existence d'un paradigme rituel ayant comme composante stable une partition du village entre invitants et invités, ces derniers étant fournisseurs de bière (Viveiro de Castro 1986, Teixeira Pinto 1989, Estival 1991). Des homologies formelles existent en ce qui concerne la syntaxe de certains orchestres alternants d'Amazonie (tagat tagat arara, tule wayâpi): en particulier le principe de concaténation de formules de longueurs différentes au sein de périodes de longueur fixe répétées ad lib. Tout ceci méritant bien sûr d'autres investigations... 


\section{BIBLIOGRAPHIE}

BEAUDET Jean-Michel, 1983, Les orchestres de clarinettes tule des Wayãpi du Haut-Oyapock. Thèse de $3^{e}$ cycle, Université Paris-X Nanterre.

ESTIVAL Jean-Pierre, 1991, « La musique instrumentale dans un rituel arara de la saison sèche (Pará, Brésil) », Journal de la Société des Américanistes LXXVII : 125-156.

IZIKOWITZ Karl Gustav, 1935, Musical and Other Sound Instruments of the South American Indians, a Comparative Ethnographical Study. Göteborg : Elanders Boktryckeri Aktiebolag. JOHNSON-LAIRD Philip Nicholas, 1991, « Jazz Improvisation: A Theory at the Computational Level », in: Representing Musical Structure, ed. by Peter Howell, Robert West, Ian Cross. London: Academic Press: 291-325.

MÜLLER R.A.P., 1985, « Abstracionismo geométrico na pintura corporal Asurini », in : Arte e corpo, pintura sobre a pele e adornos de povos indigenas brasileiros, s.l. : Ministerio da Cultura, FUNARTE : 20-25.

MÜLLER R.A.P., 1985, 1985, « Asuriní do Xingu », Revista de Antropologia (São Paulo), 27/28 : 91-113.

MÜLLER R.A.P., 1987, De como cinqüenta e duas pessoas reproduzem uma sociedade. Tese de Doutorado, São Paulo, USP.

ROUGET Gilbert, 1961, « Un chromatisme africain », L'Homme I, 3 : 30-46.

RUWET Nicolas, 1972, Langage, musique, poésie. Paris : Le Seuil.

TEIXEIRA PINTO M., 1989, Os Arara : Tempo, Espaço e Relacões Sociais em um Povo Karibe. Dissertação de mestrado, PPGAS do Museu Nacional, Universidade Federal do Rio de Janeiro.

VIVEIROS DE CASTRO E., 1985, « Bibliografia Etnológica Básica Tupi-Guarani », Revista de Antropologia (São Paulo) 27/28 : 7-24.

VIVEIROS DE CASTRO E., 1986, Arawété, os deuses canibais. Rio de Janeiro : Jorge Zahar Editor/ Anpocs.

\section{NOTES}

1. Ce n'est pas le cas pour l'aspect organologique, où l'on dispose de la somme d'Izikowitz (1935).

2. Le substantif tule désigne à la fois le complexe rituel, les pièces instrumentales et les instruments.

3. Et qui a donné lieu à une traduction du tupi en français, avec la collaboration d'une informatrice parlant le portugais.

4. Il existe plusieurs variétés de ces bouillies (de maïs) dont l'une, kaujimo'opyra, est fermentée et consommée lors du rituel tule.

5. Dont Müller (1987 : 157-160) donne une version très légèrement différente.

6. Les trois premiers par Müller, décrits dans sa thèse (1987).

7. Müller (1987: 121) classe un ensemble de rituels au sein du tule:1) le rituel des guerriers boakara, où ces derniers sont tatoués pour avoir tué un ennemi ; 2) le rituel des femmes tauwa ; 3 ) 
le rituel des morts kawara et l'initiation des jeunes kaujraõ ; 4) la danse liée à la guerre tiwagawa. En 1978 (Müller 1987), et sans doute en 1982 et 84, diverses combinaisons de ces rites ont été effectuées pendant la saison humide. Les liens entre ces rituels parfois simultanés sont loin d'être clairs, mais il n'y a pas nécessité de concomitance, comme le montre l'ethnographie du tule de 1989 (et aussi, par exemple, le fait que la danse tiwagawa ait été effectuée seule en 1990).

8. Fundaço Nacional do Indio, organisme d'État qui contrôle les populations amérindiennes et leurs terres.

9. Il semble que lorsque la société Asurini, dans les années 1930-1940, était dans un état démographique relativement stable, sa population [environ 150 personnes d'après les estimations de R. Müller (1987 : 51)] se répartissait en différents villages, chacun d'entre eux représentant un groupe local, correspondant aux groupes résidentiels d'aujourd'hui. Les informations recueillies en 1989 permettent d'avancer que ces groupes locaux restaient fortement liés entre eux, en particulier au niveau cérémoniel lors du tule, et que les relations sociales entre les groupes oscillaient entre l'alliance et le conflit ouvert suivant les moments.

10. Toutes les combinaisons sont possibles, selon les vœux du maître cérémoniel et les disponibilités de chacun.

11. Ce qui n'est pourtant pas si simple d'un point de vue syntaxique, comme nous le verrons plus loin.

12. Les clarinettes de la musique classique occidentale "quintoient», c'est-à-dire qu'une pression plus forte de la colonne d'air provoque l'émission du $3^{\mathrm{e}}$ harmonique, qui est la quinte de l'octave au dessus du fondamental.

13. Dans un cas où des parties furent doublées, plusieurs tulepy jouant mytera, j'ai même pu observer que l'un de ces tulemytera, sans doute désaccordé, était plus grave que ywyra.

14. C'est-à-dire que les harmoniques ont une fréquence dont la valeur est un multiple entier de la fréquence du fondamental.

15. Il s'agit de huit enregistrements en situation, à divers moments du rituel, où j'avais noté qu'il y avait eu accordage d'un ou plusieurs instruments. Il est clair que seule une partie des accordages effectivement réalisés ont été enregistrés.

16. C'est-à-dire que l'on peut segmenter le continuum sonore selon un critère explicite, par exemple la présence de silences ou la répétition de motifs identifiables.

17. Nous avons également effectué un travail de synthèse, à partir d'un ensemble de règles implémentées sur ordinateur, dans le même esprit que pour les flûtes de pan arara (Estival 1991).

18. Cette non-unicité de la segmentation, que l'on ne rappelle que rarement, représente un des handicaps des analyses purement formelles et «en soi » de systèmes musicaux tirés de leur contexte.

19. La partie tahira est écrite en dessous de ywyra, suivant ainsi l'ordre de jeu des musiciens.

20. En essayant de «faire parler » les informateurs sur leur pratique, il m'est apparu que c'est à ce jeu simultané que se référaient de préférence les Asurini pour caractériser les tule. La pertinence de cette distinction a été montrée lors d'une expérimentation sur le terrain effectuée lors d'une mission en 1990.

21. Plus d'une soixantaine de pièces tule ont été transcrites et analysées, et sont traitées dans un travail plus important, à paraître ultérieurement. 


\section{RÉSUMÉS}

The author focusses on the specific musical aspects of a form of instrumental polyphony in the Brazilian Amazon, namely the tule of the Asurini, taken as an identifiable sound product. After referring briefly to the global social and ritual context, he describes the instruments, the scales, the timbres and the musical structure of this instrumental tradition. He addresses in particular the principles of segmentation, the question of taxonomy, and the interaction between dancing and sound production.

\section{AUTEUR}

\section{JEAN-PIERRE ESTIVAL}

Jean-Pierre Estival, 35 ans, est inspecteur principal de la musique, chargé des musiques traditionnelles, à la Direction de la Musique et de la Danse (Ministère de la Culture, France). Après des études de mathématiques/informatique et des études musicales de contrebasse, il se consacre à l'ethnomusicologie : musique arménienne, puis terrain amazonien (moyen-Xingu/basIriri, Parà, Brésil) en 1987, 1989/1990 chez les Arara et les Asurini do Xingu. Il a enseigné l'informatique appliquée à l'ethnologie à l'Université Paris X (1987-1990). 